\title{
Search for charged Higgs bosons: combined results using LEP data
}

\author{
ALEPH Collaboration, DELPHI Collaboration, L3 Collaboration, OPAL Collaboration, The LEP working group \\ for Higgs boson searches
}

CERN, 1211 Geneva 23, Switzerland

Received: 30 January 2013 / Revised: 29 March 2013 / Published online: 3 July 2013

(C) The Author(s) 2013. This article is published with open access at Springerlink.com

\begin{abstract}
The four LEP collaborations, ALEPH, DELPHI, L3 and OPAL, have searched for pair-produced charged Higgs bosons in the framework of Two Higgs Doublet Models (2HDMs). The data of the four experiments have been statistically combined. The results are interpreted within the 2HDM for Type I and Type II benchmark scenarios. No statistically significant excess has been observed when compared to the Standard Model background prediction, and the combined LEP data exclude large regions of the model parameter space. Charged Higgs bosons with mass below $80 \mathrm{GeV} / c^{2}$ (Type II scenario) or $72.5 \mathrm{GeV} / c^{2}$ (Type I scenario, for pseudo-scalar masses above $12 \mathrm{GeV} / c^{2}$ ) are excluded at the $95 \%$ confidence level.
\end{abstract}

\section{Introduction}

A charged Higgs boson appears in many extensions of the Higgs sector beyond the Standard Model (SM). Indeed, its discovery would signal unambiguously that the Higgs-like particle recently discovered at LHC $[1,2]$ is not the SM neutral Higgs boson. It is thus of great interest to search for a charged Higgs boson.

More than twelve years after the end of data-taking at LEP, it is important to add to the LEP legacy the outcome of the searches for a charged Higgs boson. In fact, the charged Higgs boson searches at a lepton collider are significantly less model-dependent than the corresponding searches at hadron colliders, due to the very simple production mechanism.

Since the previous communication by the LEP working group for Higgs boson searches (LEPHWG) on charged

The authors are listed in Refs. [4, 5, 7, 8, 10]. Corresponding author: Pierre Lutz belongs to the DELPHI Collaboration and the LEP working group for Higgs boson searches; e-mail: lutz@in2p3.fr. Postal address: DSM/IRFU/SPP, CEA Saclay, 91191 Gif sur Yvette Cedex, France
Higgs boson, in 2001 [3], the LEP experiments have published their final results on these searches and have, in some cases, also added searches for new final states not previously considered. The four LEP collaborations have searched for charged Higgs bosons in the framework of Two Higgs Doublet Models (2HDMs). Based on the final results obtained by ALEPH [4], DELPHI [5, 6], L3 [7] and OPAL [8, 9], the LEPHWG has performed a statistical combination of the data taken at center-of-mass energies, $\sqrt{s}$, from $183 \mathrm{GeV}$ to $209 \mathrm{GeV}$. The total luminosity used in this combination is $2.6 \mathrm{fb}^{-1}$.

In 2HDMs [11-14], there are five physical Higgs bosons: the $\mathrm{CP}$-even $\mathrm{h}$ and $\mathrm{H}$, the $\mathrm{CP}$-odd $\mathrm{A}$ and the charged Higgs bosons, $\mathrm{H}^{ \pm}$. The charged Higgs couplings to the photon and the $\mathrm{Z}$ boson are completely specified in terms of the electric charge and the weak mixing angle, $\theta_{\mathrm{W}}$, and therefore, at tree level, the production cross-section depends only on the charged Higgs boson mass. Higgs bosons couple proportionally to the particle mass and therefore decay preferentially to heavy particles, but the precise branching ratios may vary significantly depending on the model. Two scenarios are considered in this paper. The first one effectively allows the charged Higgs boson to decay to fermions only, which is the case in type II 2HDM for not too small values of $m_{\mathrm{A}}$ (the neutral CP-odd A boson mass) or $\tan \beta$ (the ratio of the two Higgs doublet vacuum expectation values). In this model the isospin $+\frac{1}{2}$ fermion-couplings to the charged Higgs boson are proportional to $1 / \tan \beta$, while the isospin $-\frac{1}{2}$ fermion-couplings are proportional to $\tan \beta$. This scenario is treated in Sect. 2. In the second scenario, type I 2HDM, all fermions couple proportionally to $1 / \tan \beta$. Consequently, the second scenario effectively allows the charged Higgs boson to also decay into gauge (possibly off-shell) bosons and Higgs bosons (see Sect. 3).

Pair-production of charged Higgs bosons via s-channel exchange of a $Z^{0}$ boson would modify the decay width of the $\mathrm{Z}^{0}$ boson. Therefore electroweak precision measurements 
set indirect bounds on the mass of the charged Higgs boson regardless of its decay branching ratios. The difference between the measured decay width of the $\mathrm{Z}^{0}\left(\Gamma_{\mathrm{Z}}\right)$ and the prediction from the SM sets a limit on any non-standard (non $\mathrm{SM}$ ) contribution to $Z^{0}$ decay. The $Z^{0}$ decay width has been measured precisely during the first phase of LEP (LEP-1). The final LEP result [15] set the limit $\Gamma_{\text {nonSM }}<2.9 \mathrm{MeV} / c^{2}$ (95\% C.L.), which translates to $m_{\mathrm{H}^{ \pm}}>39.6 \mathrm{GeV} / c^{2}(95 \%$ C.L.). Direct searches during the LEP-1 period set a lower bound for the charged Higgs boson mass at $44.1 \mathrm{GeV} / c^{2}$ at $95 \%$ C.L. for type II 2HDM [16-19]. The combination in this paper is performed for charged Higgs boson masses of $43 \mathrm{GeV} / c^{2}$ or larger, since the region below $43 \mathrm{GeV} / c^{2}$ has been covered by individual experiments.

For this combination of data, the cross-sections (and branching ratios for type II $2 \mathrm{HDM}$ ) are calculated within the HZHA program package [20], and the branching ratios of the charged Higgs boson in type I 2HDM are taken from Ref. [21].

The input from the four experiments [4-9] which is used in the combination procedure is provided on a channel-bychannel basis. The word "channel" designates any subset of the data where a Higgs boson search has been carried out. Table 1 shows a summary of all channels available for this combination. It amounts to 22 channels from ALEPH, 43 from DELPHI, 12 from L3 and 45 from OPAL.

Each experiment generated and simulated the detailed detector response in Monte Carlo event samples for the Higgs signal and the various background processes, at centerof-mass energies of 183, 189, 192, 196, 200, 202, 204, 206, 208 and $209 \mathrm{GeV}$ to estimate background and signal contributions in the data collected between 1997 and

Table 1 Overview of the searches for charged Higgs bosons performed by the four LEP experiments, whose results are used in this combination. Where relevant, $m_{\mathrm{A}}$ varies from $2 m_{b}$ to $m_{\mathrm{H}^{ \pm}}$. Each experiment analyzed typically around $650 \mathrm{pb}^{-1}$ of data

\begin{tabular}{|c|c|c|c|}
\hline Expt (ref.) & Final state & $\begin{array}{l}\sqrt{s} \\
(\mathrm{GeV})\end{array}$ & $\begin{array}{l}m_{\mathrm{H}^{ \pm}} \text {range } \\
\left(\mathrm{GeV} / c^{2}\right)\end{array}$ \\
\hline \multirow[t]{3}{*}{ ALEPH [4] } & $\mathrm{H}^{+} \mathrm{H}^{-} \rightarrow \csc \bar{s}$ & 189-209 & $45-100$ \\
\hline & $\mathrm{H}^{+} \mathrm{H}^{-} \rightarrow \operatorname{cs} \tau \nu$ & 189-209 & $55-100$ \\
\hline & $\mathrm{H}^{+} \mathrm{H}^{-} \rightarrow \tau \nu \tau \nu$ & 189-209 & $45-100$ \\
\hline \multirow[t]{5}{*}{ DELPHI $[5,6]$} & $\mathrm{H}^{+} \mathrm{H}^{-} \rightarrow \mathrm{cs} \overline{\mathrm{c}} \mathrm{s}$ & $183-209$ & $40-100$ \\
\hline & $\mathrm{H}^{+} \mathrm{H}^{-} \rightarrow \operatorname{cs} \tau \nu$ & $183-209$ & $40-100$ \\
\hline & $\mathrm{H}^{+} \mathrm{H}^{-} \rightarrow \tau \nu \tau \nu$ & $183-209$ & $40-100$ \\
\hline & $\mathrm{H}^{+} \mathrm{H}^{-} \rightarrow \mathrm{W}^{*} \mathrm{~A} \tau \nu$ & 189-209 & $40-100$ \\
\hline & $\mathrm{H}^{+} \mathrm{H}^{-} \rightarrow \mathrm{W}^{*} \mathrm{AW}^{*} \mathrm{~A}$ & $189-209$ & $40-100$ \\
\hline \multirow[t]{3}{*}{ L3 [7] } & $\mathrm{H}^{+} \mathrm{H}^{-} \rightarrow \mathrm{cs} \bar{c} \mathrm{~s}$ & $183-209$ & $50-100$ \\
\hline & $\mathrm{H}^{+} \mathrm{H}^{-} \rightarrow \operatorname{cs} \tau \nu$ & $183-209$ & $50-100$ \\
\hline & $\mathrm{H}^{+} \mathrm{H}^{-} \rightarrow \tau \nu \tau \nu$ & $183-209$ & $50-100$ \\
\hline \multirow[t]{5}{*}{ OPAL $[8,9]$} & $\mathrm{H}^{+} \mathrm{H}^{-} \rightarrow \mathrm{cs} \overline{\mathrm{c}} \mathrm{s}$ & $183-209$ & $40-100$ \\
\hline & $\mathrm{H}^{+} \mathrm{H}^{-} \rightarrow \mathrm{cs} \tau \nu$ & $183-209$ & $40-100$ \\
\hline & $\mathrm{H}^{+} \mathrm{H}^{-} \rightarrow \tau \nu \tau \nu$ & $183-209$ & $45-100$ \\
\hline & $\mathrm{H}^{+} \mathrm{H}^{-} \rightarrow \mathrm{W}^{*} \mathrm{~A} \tau \nu$ & 189-209 & $40-95$ \\
\hline & $\mathrm{H}^{+} \mathrm{H}^{-} \rightarrow \mathrm{W}^{*} \mathrm{AW}^{*} \mathrm{~A}$ & 189-209 & $40-95$ \\
\hline
\end{tabular}

2000. Particular care has been taken when simulating the four-fermion background, especially from W-pair background, using the most advanced codes available at that time. ALEPH used KORALW [22] as the generator and RACOONWW [23] and YFSWW [24] for the cross-section calculation, while DELPHI used WPHACT [25], L3 YFSWW and OPAL GRC4F [26] and KORALW. Other generators were used for systematic studies. Furthermore, each of the four experiments used different values for the $\mathrm{W}$ mass in these background simulations (respectively 80.45, 80.40, 80.356 and $80.33 \mathrm{GeV} / c^{2}$ for ALEPH, DELPHI, L3 and OPAL), while the LEP combined measured value is $80.376 \pm 0.033 \mathrm{GeV} / c^{2}$ [27], thus introducing an additional source of systematic uncertainty due to the broadening of the $\mathrm{W}$ peak when adding the four background simulations.

The statistical procedure adopted for the combination of the data and the precise definitions of the confidence levels $\mathrm{CL}_{b}, \mathrm{CL}_{s}, \mathrm{CL}_{s+b}$ by which the search results are expressed, have been described previously [28]. The main sources of systematic uncertainty affecting the signal and background predictions are included, using an extension of the method of Cousins and Highland [29] where the $p$-values are averaged over a large ensemble of Monte Carlo experiments. The correlations between search channels, LEP collision energies and individual experiments have not been taken into account, but these correlations are estimated to have only small effects, about $500 \mathrm{MeV} / \mathrm{c}^{2}$, to the final results.

\section{Combined searches in the framework of type II 2HDM}

In type II 2HDM, one Higgs doublet couples to up-type fermions and the other to down-type fermions. The Higgs sector of the Minimal Supersymmetric Standard Model (MSSM) is a particular case of such models. In the MSSM, at tree-level, the $\mathrm{H}^{ \pm}$is constrained to be heavier than the $\mathrm{W}$ boson and the radiative corrections to the charged Higgs mass are positive, except for very specific parameter choices. Thus, experimentally finding evidence of a charged Higgs boson with mass below the $\mathrm{W}$ boson mass would set very strong constraints on the MSSM parameters. However, in the following we will concentrate on the general type II 2HDM without any supersymmetric assumptions. Results on the search for neutral MSSM Higgs bosons can be found in [30].

For the charged Higgs masses accessible at LEP energies, the decays into $\tau^{+} v_{\tau}$ and $\mathrm{cs}$ (and their charge conjugates) are expected to dominate. The searches are carried out under the assumption that the two decays $\mathrm{H}^{+} \rightarrow c \overline{\mathrm{s}}$ and $\mathrm{H}^{+} \rightarrow \tau^{+} v$ exhaust the $\mathrm{H}^{+}$decay width, but the relative branching ratio is free. This assumption is valid as long as $m_{\mathrm{A}}$ is larger than $60 \mathrm{GeV} / c^{2}$ (MSSM case) or $\tan \beta$ is 
larger than a few units. Thus, the searches encompass the following $\mathrm{H}^{+} \mathrm{H}^{-}$final states: $(\mathrm{cs})(\overline{\mathrm{cs}}),\left(\tau^{+} v\right)\left(\tau^{-} \bar{v}\right)$ and the mixed mode $(\mathrm{c} \bar{s})\left(\tau^{-} \bar{v}\right)$ or $(\overline{\mathrm{c} s})\left(\tau^{+} \nu\right)$. The combined search results are presented as a function of the branching ratio $\operatorname{Br}\left(\mathrm{H}^{+} \rightarrow \tau^{+} v\right)$.

Details of the searches done by the individual experiments can be found in Refs. [4-9]. Two features in these analyses are worth noting: the main background is $\mathrm{W}$ pair production, which is partly irreducible, and the reconstructed mass is one of the discriminant variables used in the final hypothesis testing in the two channels where this is relevant (mixed and hadronic channels). The results from the four LEP experiments are summarized in Table 2, together with the $95 \%$ C.L. observed and median expected lower limits on the charged Higgs boson mass. The mass

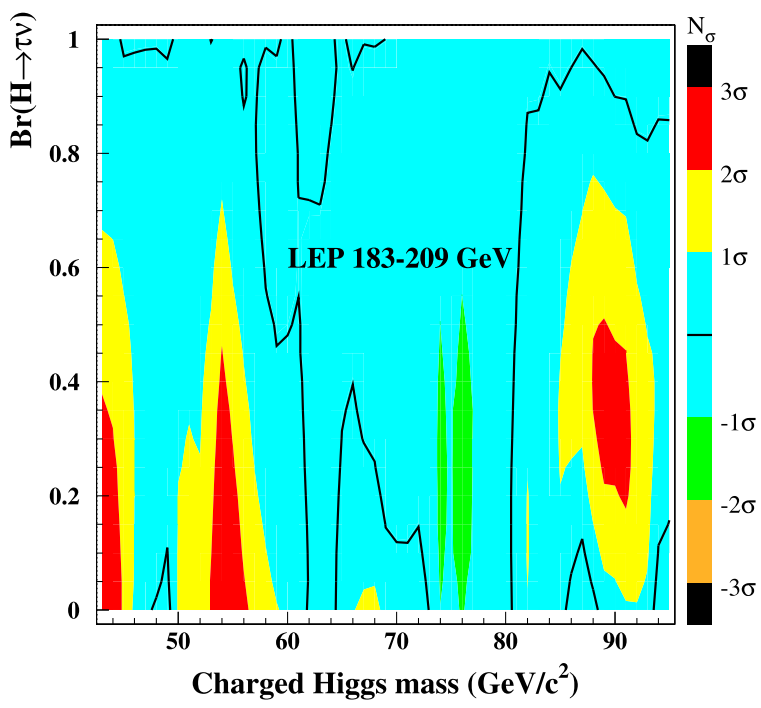

Fig. 1 Type II 2HDM: contours based on the observed p-values $\mathrm{CL}_{b}$ as a function of $m_{\mathrm{H}^{ \pm}}$and the branching ratio $\mathrm{Br}\left(\mathrm{H}^{+} \rightarrow \tau^{+} \nu\right)$, indicating the statistical significance, $N_{\sigma}$, of local departures from the background expectation. The black solid line indicates the change of sign of this significance, i.e. where there is a transition from excess to deficit limits are quoted separately for $\operatorname{Br}\left(\mathrm{H}^{+} \rightarrow \tau^{+} \nu\right)=0,1$, and independently of the charged Higgs decay.

In a first step, the statistical combination software was run separately on the data provided by the four collaborations and the results compared to the published results of each. The differences between this check and the published results (of the order of $\pm 200 \mathrm{MeV} / c^{2}$ in the limits) reflect the differences between the statistical methods used by the four collaborations. The biggest difference has been found for the expected limit from OPAL at $\mathrm{Br}\left(\mathrm{H}^{+} \rightarrow \tau^{+} v\right)=1$ and amounts to $600 \mathrm{MeV} / c^{2}$. This difference is compatible in size with the estimated effect of about $500 \mathrm{MeV} / c^{2}$ of not taking into account the correlations between systematic uncertainties. All mass limits have thus been rounded down to the nearest half a $\mathrm{GeV} / c^{2}$.

Combining the results from the four experiments, a scan in the branching ratio $\mathrm{Br}\left(\mathrm{H}^{+} \rightarrow \tau^{+} \nu\right)$ versus charged Higgs boson mass plane has been performed, and the limit-setting procedure was repeated for each scan point. This twodimensional scan was performed with the following ranges and steps: $m_{\mathrm{H}^{ \pm}}$from 43 to $95 \mathrm{GeV} / c^{2}$ with $1 \mathrm{GeV} / c^{2}$ steps, and $\mathrm{Br}\left(\mathrm{H}^{+} \rightarrow \tau^{+} \nu\right)$ from 0 to 1 with 0.05 steps.

Figure 1 shows the observed background confidence level $\mathrm{CL}_{b}$ as a function of $m_{\mathrm{H}^{ \pm}}$and $\operatorname{Br}\left(\mathrm{H}^{+} \rightarrow \tau^{+} \nu\right)$. The observed confidence level is everywhere within $\pm 2 \sigma$ of the background prediction, except for three small regions, as shown in Fig. 1. Such regions result from the combination of small excesses, as compared to the background expectation, observed by two or three experiments. The first two mass regions, around 43 and $55 \mathrm{GeV} / c^{2}$, are due to a slight excess of data in the hadronic channel, and the third one, around $90 \mathrm{GeV} / c^{2}$, arises from an excess of events in the mixed channel. Table 3 gives the combined $\mathrm{CL}_{b}$ together with the values from each experiment for these three domains (the values chosen for $\operatorname{Br}\left(\mathrm{H}^{+} \rightarrow \tau^{+} \nu\right)$ are given in the second column).

The combined results for the Type II 2HDM are summarized in Fig. 2, which shows the expected median and observed mass limits, while the contribution of each of the
Table 2 Individual search results for the $\mathrm{e}^{+} \mathrm{e}^{-} \rightarrow \mathrm{H}^{+} \mathrm{H}^{-}$ fermionic final states. All limits are given at the $95 \%$ C.L. The OPAL selection is mass-dependent; the numbers of events given here are for $m_{\mathrm{H}^{ \pm}}=80 \mathrm{GeV} / c^{2}$

\begin{tabular}{|c|c|c|c|c|}
\hline Experiment & ALEPH [4] & DELPHI [5] & L3 [7] & OPAL [8] \\
\hline Total Int. luminosity $\left(\mathrm{pb}^{-1}\right)$ & 630 & 620 & 685 & 670 \\
\hline Final states & \multicolumn{4}{|c|}{ Number of expected/observed events } \\
\hline$(\mathrm{c} \overline{\mathrm{s}})(\overline{\mathrm{cs}})$ & $2806.0 / 2742$ & $2179.3 / 2179$ & $2473.8 / 2578$ & $1501.4 / 1471$ \\
\hline$(\mathrm{c} \overline{\mathrm{s}})\left(\tau^{-} \bar{v}\right)$ & $289.3 / 280$ & $1122.8 / 1129$ & $494.5 / 470$ & $526.3 / 569$ \\
\hline$\left(\tau^{+} \nu\right)\left(\tau^{-} \bar{v}\right)$ & $39.8 / 45$ & $73.6 / 66$ & $149.8 / 147$ & $1103.4 / 1110$ \\
\hline Sum of all channels & $3135.1 / 3067$ & $3375.7 / 3374$ & $3118.1 / 3195$ & $3131.1 / 3150$ \\
\hline \multicolumn{5}{|c|}{$\begin{array}{l}\text { Mass limits in } \mathrm{GeV} / c^{2} \\
\text { Expected(median)/observed limit }\end{array}$} \\
\hline $\operatorname{Br}\left(\mathrm{H}^{+} \rightarrow \tau^{+} \nu\right)=0$ & $78.2 / 80.4$ & $77.7 / 77.8$ & $76.8 / 76.6$ & $77.2 / 76.5$ \\
\hline $\mathrm{Br}\left(\mathrm{H}^{+} \rightarrow \tau^{+} v\right)=1$ & $89.2 / 87.8$ & $88.9 / 90.1$ & $84.3 / 83.7$ & $89.2 / 91.3$ \\
\hline any $\mathrm{Br}\left(\mathrm{H}^{+} \rightarrow \tau^{+} \nu\right)$ & $77.1 / 79.3$ & $76.3 / 74.4$ & $75.7 / 76.4$ & $75.6 / 76.3$ \\
\hline
\end{tabular}


Table 3 Combined and individual $\mathrm{CL}_{b}$ values for the three mass points with a deviation from expectation larger than $2 \sigma$. All values, obtained with the statistical procedure of the overall combination, compare well to those published by the experiments. (*) ALEPH and L3 did not provide inputs for this mass

\begin{tabular}{lllllll}
\hline $\begin{array}{l}m_{\mathrm{H}^{ \pm}} \\
\left(\mathrm{GeV} / c^{2}\right)\end{array}$ & $\mathrm{Br}\left(\mathrm{H}^{+} \rightarrow \tau^{+} v\right)$ & combined $\mathrm{CL}_{b}$ & ALEPH CL & DELPHI CL & L3 CL $C_{b}$ & OPAL CL $_{b}$ \\
\hline 43 & 0.0 & 0.998 & $(*)$ & 0.99 & $(*)$ & 0.96 \\
55 & 0.0 & 0.997 & 0.75 & 0.96 & 0.96 & 0.94 \\
89 & 0.35 & 0.988 & 0.98 & 0.63 & 0.88 & 0.80 \\
\hline
\end{tabular}

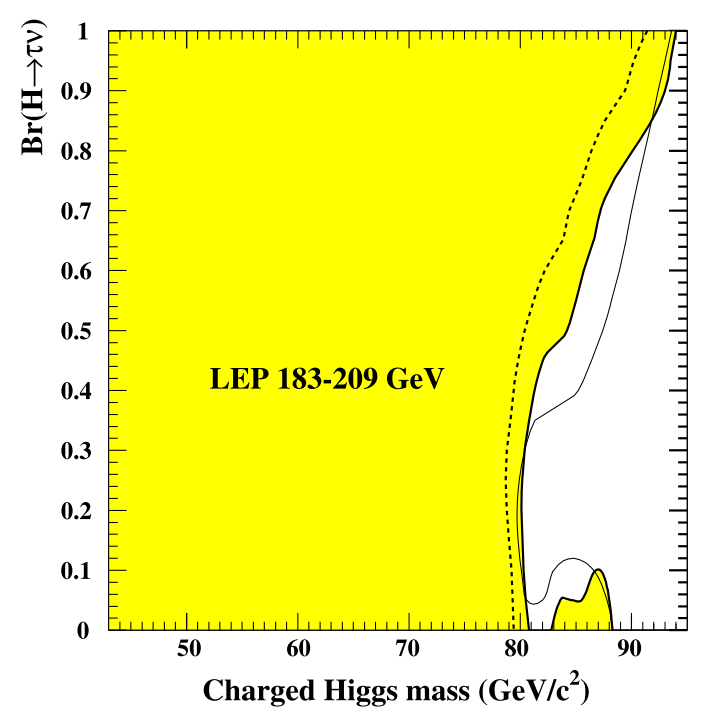

Fig. 2 Type II 2HDM: excluded regions in the $\operatorname{Br}\left(\mathrm{H}^{+} \rightarrow \tau^{+} v\right)$ vs $m_{\mathrm{H}^{ \pm}}$plane, based on the combined data collected by the four LEP experiments at center-of-mass energies from 183 to $209 \mathrm{GeV}$. The shaded area is excluded at the $95 \%$ or higher C.L. The expected exclusion limit (at the $95 \%$ C.L.) is indicated by the thin solid line and the thick dotted line inside the shaded area is the observed limit at the $99.7 \%$ C.L.

three decay channels to the overall limit is presented in Fig. 3. It is worth noting that:

- The purely leptonic channel alone excludes charged Higgs masses above the $\mathrm{W}$ mass, down to $\mathrm{Br}\left(\mathrm{H}^{+} \rightarrow \tau^{+} v\right)$ around 0.45 . In this channel, the mass of the Higgs boson cannot be reconstructed, due to the presence of two neutrinos in the final state. As a consequence, the $\mathrm{W}$ boson pair background is diluted and the analysis is sensitive up to $\sqrt{s} / 2$. The limit drops rapidly for $\mathrm{Br}\left(\mathrm{H}^{+} \rightarrow \tau^{+} v\right)$ below 0.45 , due to a rapid decrease of the signal rate in this final state.

- The mixed channel alone cannot exclude charged Higgs mass values up to the $\mathrm{W}$ mass, even when it contributes maximally, for $\operatorname{Br}\left(\mathrm{H}^{+} \rightarrow \tau^{+} v\right)=0.5$. For this value, the observed limit is only slightly above $79 \mathrm{GeV} / c^{2}$, due to the large $\mathrm{e}^{+} \mathrm{e}^{-} \rightarrow \mathrm{W}^{+} \mathrm{W}^{-}$background. This channel has the best coverage in terms of $\mathrm{Br}\left(\mathrm{H}^{+} \rightarrow \tau^{+} v\right)$, as shown in Fig. 3.

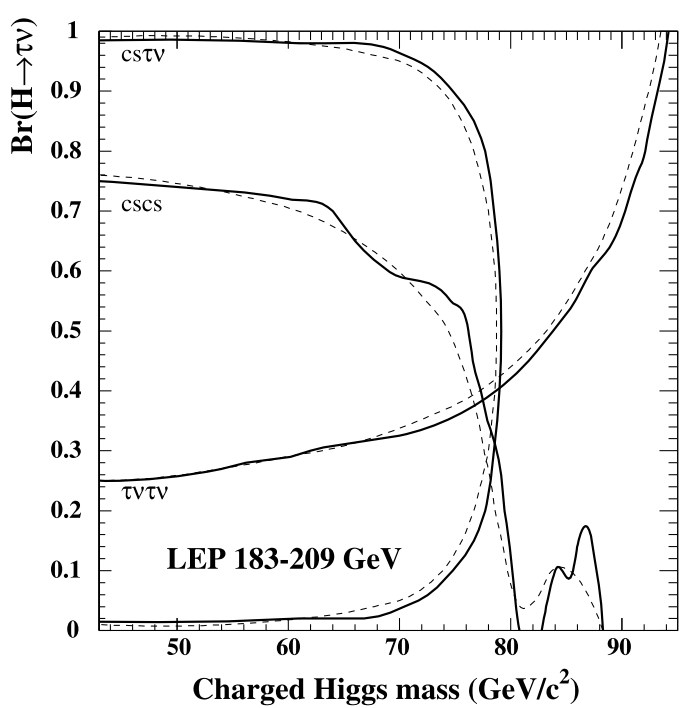

Fig. 3 Type II 2HDM: regions in the $\mathrm{Br}\left(\mathrm{H}^{+} \rightarrow \tau^{+} v\right)$ vs $m_{\mathrm{H}^{ \pm}}$plane excluded at the $95 \%$ or higher C.L., based on the combined data collected by the four LEP experiments at center-of-mass energies from 183 to $209 \mathrm{GeV}$, for each of the three decay channels separately. The solid (dashed) lines are the observed (expected) limits

- The hadronic channel is the most difficult one; for masses close to the $\mathrm{W}$ mass, the sensitivity is reduced due to the large $\mathrm{e}^{+} \mathrm{e}^{-} \rightarrow \mathrm{W}^{+} \mathrm{W}^{-}$background. The sensitivity at higher masses is improved (a gain of $10 \mathrm{GeV} / c^{2}$ on the expected limit), and the observed limit as well (note the excluded "island" at $\operatorname{Br}\left(\mathrm{H}^{+} \rightarrow \tau^{+} v\right)$ close to zero) with respect to the results of individual experiments.

- The difference between the expected and observed limit seen in Fig. 2 for $\operatorname{Br}\left(\mathrm{H}^{+} \rightarrow \tau^{+} v\right)$ from 0.35 to 0.85 results from the excess of observed events already mentioned (see Fig. 1) in the mixed channel above $m_{\mathrm{H}^{ \pm}}=$ $84 \mathrm{GeV} / c^{2}$.

The combined $95 \%$ C.L. $m_{\mathrm{H}^{ \pm}}$lower limits are listed in Table 4 for $\mathrm{Br}\left(\mathrm{H}^{+} \rightarrow \tau^{+} v\right)=0,1$, together with the limit that is independent of the fermionic decay mode. Taking the lowest of the observed limits from Table 4, we quote a $95 \%$ C.L. lower bound of $80 \mathrm{GeV} / c^{2}$ for the mass of the charged Higgs boson in type II 2HDM under the assumption of pure fermionic decays of the charged Higgs boson. Thus the hy- 

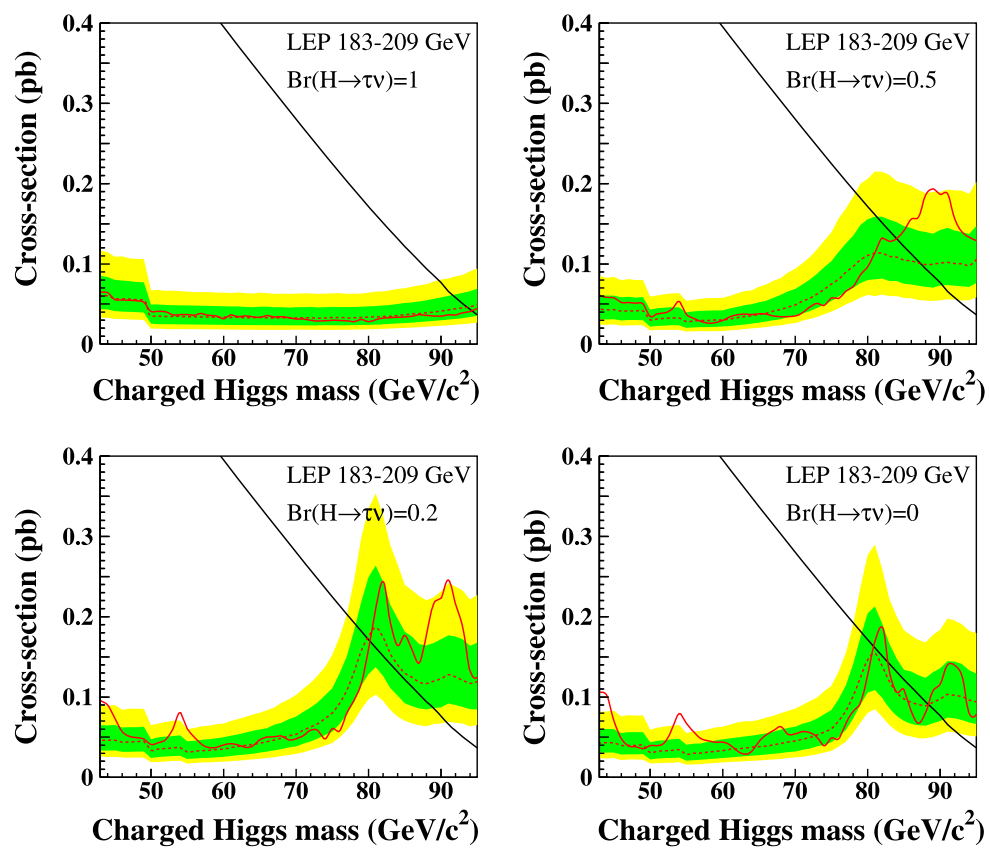

Fig. 4 Type II 2HDM: the $95 \%$ C.L. upper limits on the production cross-section as a function of $m_{\mathrm{H}^{ \pm}}$for four different values of the branching ratio $\mathrm{Br}\left(\mathrm{H}^{+} \rightarrow \tau^{+} \nu\right)$, combining the data collected by the four LEP experiments at center-of-mass energies from 183 to $209 \mathrm{GeV}$. The solid lines represent the observed exclusion limits, while the expected exclusion limits are indicated by the dashed lines. The shaded

Table 4 The combined $95 \%$ C.L. lower bounds on the mass of the charged Higgs boson (in $\mathrm{GeV} / c^{2}$ ), expected and observed, for fixed values of the branching ratio $\operatorname{Br}\left(\mathrm{H}^{+} \rightarrow \tau^{+} \nu\right)$ and for any $\operatorname{Br}\left(\mathrm{H}^{+} \rightarrow \tau^{+} v\right)$. All mass limits have been rounded down to the nearest half a GeV $/ c^{2}$ to take into account the effect of neglecting the correlations between systematic uncertainties. (*) The interval from 83 to 88 $\mathrm{GeV} / c^{2}$ is also excluded at the $95 \%$ C.L.

\begin{tabular}{lll}
\hline & Expected limit (median) & Observed limit \\
\hline $\operatorname{Br}\left(\mathrm{H}^{+} \rightarrow \tau^{+} \nu\right)=0$ & 88 & $80.5\left(^{*}\right)$ \\
$\operatorname{Br}\left(\mathrm{H}^{+} \rightarrow \tau^{+} \nu\right)=1$ & 93.5 & 94 \\
Any $\operatorname{Br}\left(\mathrm{H}^{+} \rightarrow \tau^{+} \nu\right)$ & 79.5 & 80 \\
\hline
\end{tabular}

pothesis of a charged Higgs boson degenerate in mass with the $\mathrm{W}$ boson is not excluded at the $95 \%$ confidence level with LEP data. The limits around the $\mathrm{W}$ mass are very sensitive to the modeling of the $\mathrm{W}$ pairs background. Taking the uncertainties in the background modeling (including the uncertainty due to different simulated $\mathrm{W}$ masses) into account results in a downward shift of these limits by 600 and $500 \mathrm{MeV} / c^{2}$ for $\mathrm{Br}\left(\mathrm{H}^{+} \rightarrow \tau^{+} \nu\right)=0$ and 0.5 , respectively.

Figure 4 shows the $95 \%$ C.L. upper bound on the $\mathrm{e}^{+} \mathrm{e}^{-} \rightarrow \mathrm{H}^{+} \mathrm{H}^{-}$cross-section (with $\pm 1 \sigma$ and $\pm 2 \sigma$ bands) for four values of $\mathrm{Br}\left(\mathrm{H}^{+} \rightarrow \tau^{+} \nu\right.$ ), namely 1, 0.5, 0.2 (which corresponds to the weakest limit) and 0 . The thick black curve is the $2 \mathrm{HDM}$ tree-level prediction for that crosssection. bands represent the $\pm 1 \sigma$ and $\pm 2 \sigma$ excursions around the expected limits. The intersections of the curves (solid or dashed) with the thick line showing the theoretical (tree-level) charged Higgs cross-section represent the (observed or expected) $95 \%$ C.L. lower limits on the charged Higgs boson mass

\section{Combined searches in 2HDM of type I}

An alternative set of models, type I 2HDMs, assume that all fermions couple to the same Higgs doublet. In this case all fermions couple proportionally to $1 / \tan \beta$ to the charged Higgs boson and fermionic decays are suppressed for medium to large $\tan \beta$ values. Consequently, if a neutral Higgs boson $\Phi$ (representing either A or the lightest CPeven scalar h) is sufficiently light, the decay to $\mathrm{W}^{*} \Phi$ can be dominant even in the range of charged Higgs masses of interest at LEP (where $\mathrm{W}^{*}$ indicates an off-shell $\mathrm{W}$ boson). While searches for a CP-even neutral Higgs boson exclude such a particle for masses below $82 \mathrm{GeV} / c^{2}$ independently of its decay [31], the existence of a light $\mathrm{CP}$-odd neutral Higgs boson, A, is not excluded by experiment [32]. Hence, the search for the process $\mathrm{H}^{ \pm} \rightarrow \mathrm{W}^{*} \mathrm{~A}$ is fully justified. Figure 5 shows the predicted branching ratios of the charged Higgs bosons for various choices of parameters of type I models. For all kinematically allowed values of the A mass, $m_{\mathrm{A}}$, the possible charged Higgs boson decays are predominantly fermionic for low $\tan \beta$ and predominantly bosonic for high $\tan \beta$. Between these two extreme cases, the branching ratios change rapidly as a function of $\tan \beta$ (between typically 0.1 and 10) and slower as a function of $m_{\mathrm{A}}$, appearing earlier in $\tan \beta$ for lower $m_{\mathrm{A}}$. The ratio between the two competing fermionic decays ( $\tau \nu$ over $\mathrm{c} \overline{\mathrm{s}})$ is almost independent of the charged Higgs boson mass (see lower part 
of the figure), as expected from the Yukawa coupling which only depends upon the masses involved.

To cover the possibility of a light A boson the final states $\mathrm{W}^{*} \mathrm{AW}^{*} \mathrm{~A}$ and $\mathrm{W}^{*} \mathrm{~A} \tau^{-} \overline{\nu_{\tau}}$ were also searched for by DELPHI [5] and OPAL [8]. The channel $\mathrm{W}^{*} \mathrm{Ac}$ s was not considered because its contribution is expected to be small for all $\tan \beta$. The A boson was searched for through its decay into two b-jets, restricting the A mass to be above $12 \mathrm{GeV} / c^{2}$. Type I models are explored through the combination of all five decay channels, namely the final states $\operatorname{cs} \bar{c} s, \operatorname{cs} \tau \nu$, $\tau^{+} \nu \tau^{-} \bar{v}, \mathrm{~W}^{*} \mathrm{AW}^{*} \mathrm{~A}$ and $\mathrm{W}^{*} \mathrm{~A} \tau^{-} \overline{\nu_{\tau}}$ (and their charge conju-
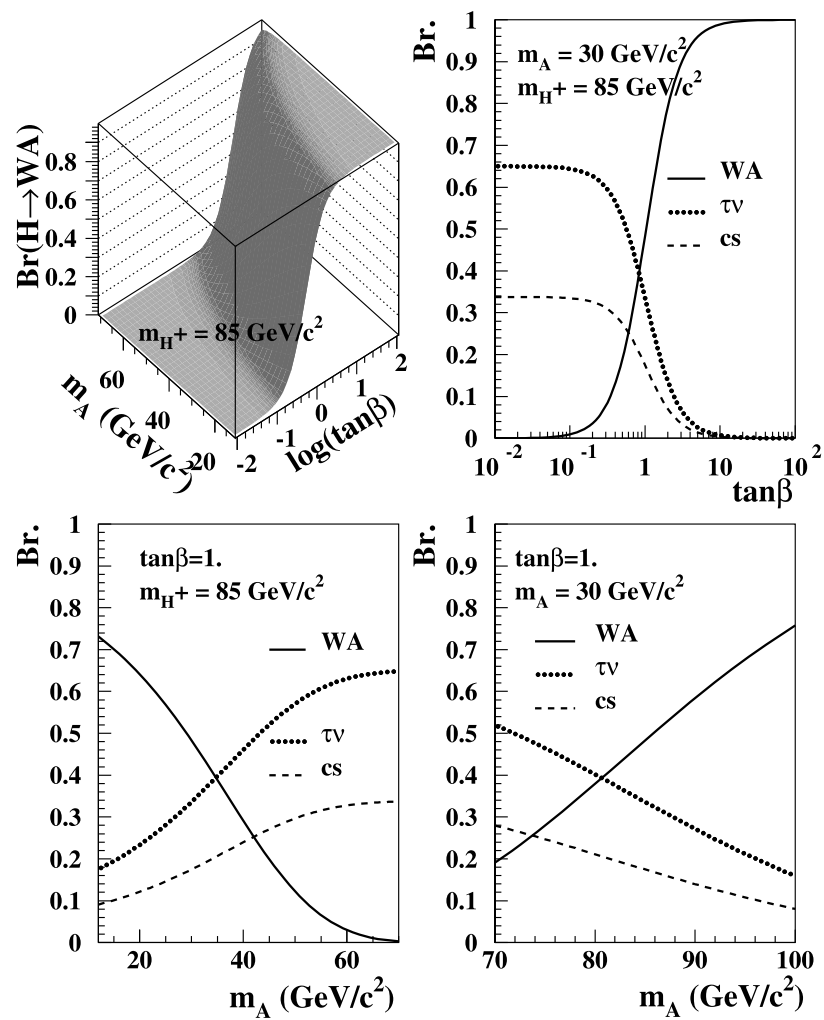

Fig. 5 Type I 2HDM: decay branching fractions as functions of the boson masses and $\tan \beta$

Table 5 Observed lower limits on the charged Higgs mass in $\mathrm{GeV} / c^{2}$ at $95 \%$ C.L. for different values of $m_{\mathrm{A}}$ (in $\mathrm{GeV} / c^{2}$ ) and $\tan \beta$. The expected median limits are shown in parentheses. The last column (last row) show the weakest limit for a fixed $\mathrm{A}$ mass and any $\tan \beta$ (for gates). The combination of the experimental search results is performed for branching ratio values predicted by the model as a function of $\tan \beta$ and $m_{\mathrm{A}}$. Where there was a possible overlap between two search channels, the one providing less expected sensitivity was ignored to avoid double counting. This is the case in the intermediate region in $\tan \beta$ for purely hadronic channels $\left(\mathrm{W}^{*} \mathrm{AW}^{*} \mathrm{~A}\right.$ and $\left.\mathrm{cs \overline {c } \mathrm { s }}\right)$ on the one hand and the semi-leptonic channels $\left(\mathrm{W}^{*} \mathrm{~A} \tau^{-} \overline{\nu_{\tau}}\right.$ and $\left.\mathrm{cs} \tau^{-} \bar{v}\right)$ on the other. A three-dimensional scan was performed with the following ranges and steps: $m_{\mathrm{H}^{ \pm}}$from 43 to $95 \mathrm{GeV} / c^{2}$ in $1 \mathrm{GeV} / c^{2}$ steps, $m_{\mathrm{A}}$ covering $12 \mathrm{GeV} / c^{2}$, then 15 to 75 $\mathrm{GeV} / c^{2}$ in $5 \mathrm{GeV} / c^{2}$ steps, and $\tan \beta$ from 0.1 to 100 in steps of 0.2 in $\log (\tan \beta)$.

Figure 6 shows the observed $\mathrm{CL}_{b}$, for four values of $m_{\mathrm{A}}$ and two values of $\tan \beta$. A slight excess for low and intermediate $\mathrm{A}$ masses in the high $\tan \beta$ region where the bosonic decays dominate is observed, resulting in observed limits generally weaker than expected (see Fig. 7). Three main features are visible in Fig. 7, two plateaux and a valley between them:

- The first plateau, at low $\tan \beta$, corresponds to the case when the fermionic channels dominate. Both expected and observed limits are above $86 \mathrm{GeV} / c^{2}$.

- The valley is somewhat of an artefact. It is due to the conservative approach of considering only the most sensitive channel when two overlapping channels contribute. The difference between expected and observed mass limits reaches $4.5 \mathrm{GeV} / c^{2}$ in the extreme case (when $\tan \beta=$ 1.6 and $m_{\mathrm{A}}=12 \mathrm{GeV} / c^{2}$ ).

- The second plateau, at high $\tan \beta$, corresponds to the case when the bosonic channels dominate. The small excess seen in Fig. 6 corresponds to a small difference between expected and observed charged Higgs mass limits, which is always less than $2.2 \mathrm{GeV} / c^{2}$.

Figure 8 shows the excluded regions at the $95 \%$ C.L. in the plane $\left(m_{\mathrm{H}^{ \pm}}, \tan \beta\right)$ for four values of $m_{\mathrm{A}}$, namely 12 , 30,50 and $70 \mathrm{GeV} / c^{2}$, together with the expected exclusion limits.

a fixed $\tan \beta$ and any $\mathrm{A}$ mass). The mass limits have been rounded down to the nearest half a $\mathrm{GeV} / c^{2}$ to take into account the effect of neglecting correlations between systematic uncertainties

\begin{tabular}{|c|c|c|c|c|c|}
\hline$m_{\mathrm{A}}$ & $\tan \beta=0.1$ & $\tan \beta=1$ & $\tan \beta=10$ & $\tan \beta=100$ & Minimum \\
\hline 12 & $86.0(86.0)$ & $73.5(77.0)$ & $83.5(86.0)$ & $84.0(86.0)$ & $72.5(77.0)$ \\
\hline 20 & $86.5(86.0)$ & $76.5(77.5)$ & $85.5(87.0)$ & $85.5(87.0)$ & 76.5 (77.5) \\
\hline 30 & $86.5(86.5)$ & $80.0(79.5)$ & $87.5(89.0)$ & $87.5(89.0)$ & $78.0(79.5)$ \\
\hline 50 & $86.5(86.5)$ & $84.0(84.0)$ & $89.0(90.0)$ & $89.5(91.0)$ & $81.0(80.5)$ \\
\hline 70 & $86.5(86.5)$ & $86.5(86.5)$ & $83.5(83.5)$ & $89.0(90.5)$ & $81.0(81.0)$ \\
\hline Minimum & $86.0(86.0)$ & $73.5(77.0)$ & $81.5(81.0)$ & $81.0(81.0)$ & $72.5(77.0)$ \\
\hline
\end{tabular}


Fig. 6 Type I 2HDM: contours based on the observed p-values $\mathrm{CL}_{b}$ as a function of $m_{\mathrm{H}^{ \pm}}$and $\tan \beta$ or $m_{\mathrm{A}}$, indicating the statistical significance, $\mathrm{N}_{\sigma}$, of local departures from the background expectation, for four values of $m_{\mathrm{A}}$ and two values of $\tan \beta$. The black solid line indicates the change of sign of this significance, i.e. where there is a transition from excess to deficit
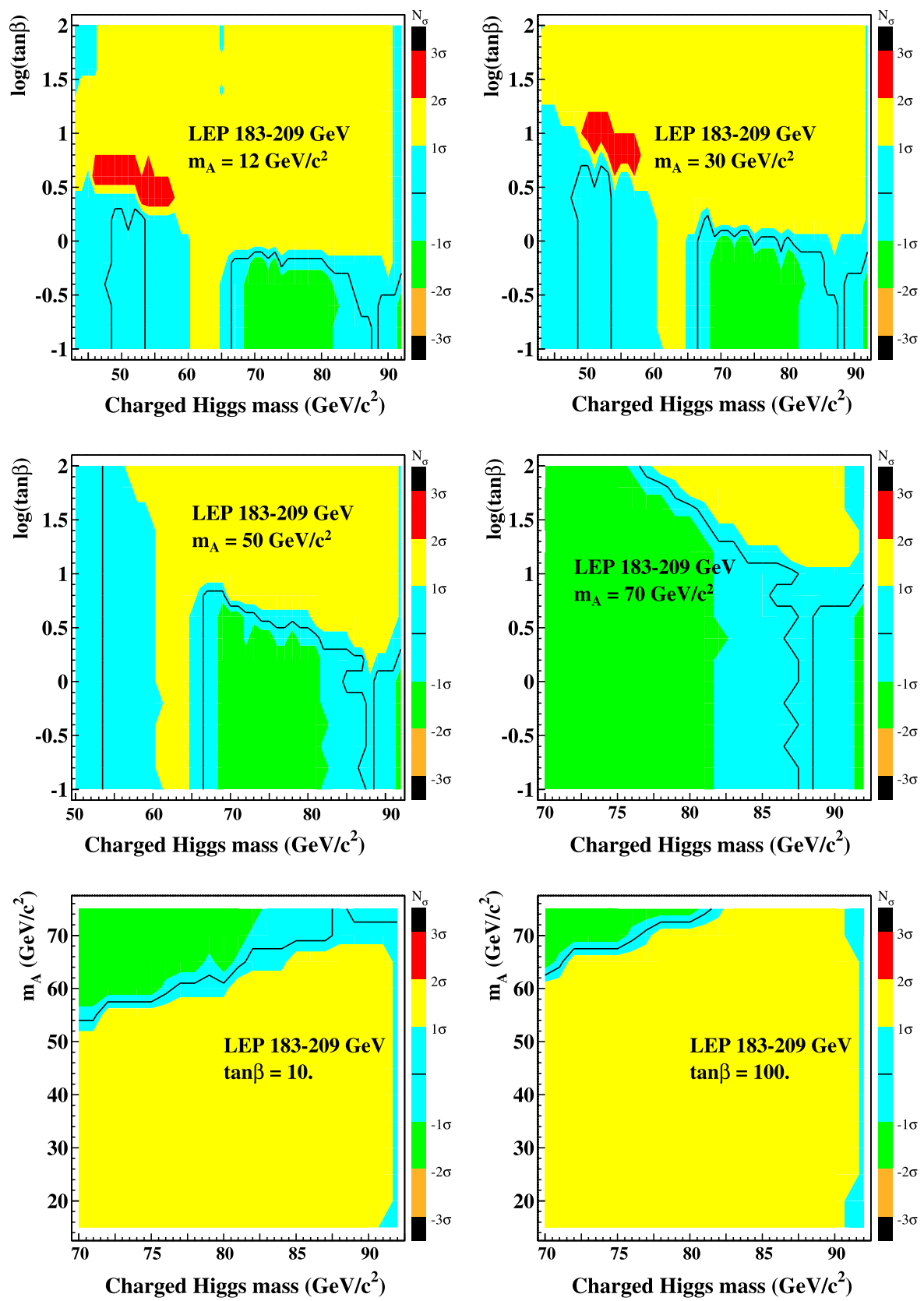

Table 5 summarizes these results. For low $\tan \beta(\tan \beta$ below 0.5 ) where the bosonic contribution is vanishingly small, the $m_{\mathrm{H}^{ \pm}}$lower limits (above $86 \mathrm{GeV} / c^{2}$ ) are almost independent of $m_{\mathrm{A}}$. On the other hand, for high $\tan \beta$ (equal to or greater than 10) where the bosonic channels dominate, the sensitivity is maximal for intermediate A masses $\left(m_{\mathrm{A}}\right.$ around $50 \mathrm{GeV} / c^{2}$ ). Outside the valley, the limit is always above $84 \mathrm{GeV} / c^{2}$. Finally, the lowest limits always correspond to the cases in the valley, thus depending both on $\tan \beta$ and $m_{\mathrm{A}}$. The lowest (observed) limit is $72.5 \mathrm{GeV} / c^{2}$, for $\tan \beta=1.6$ and $m_{\mathrm{A}}=12 \mathrm{GeV} / c^{2}$. This limit rises to $76.5 \mathrm{GeV} / c^{2}$ for $m_{\mathrm{A}}=20 \mathrm{GeV} / c^{2}$ and the difference between expectation and observation is reduced to $1 \mathrm{GeV} / c^{2}$.

\section{Summary}

The results of the searches carried out by the four LEP experiments for charged Higgs bosons have been statistically combined and interpreted in 2HDMs. No significant excess over the SM background is observed, and the exclusion limits are extended by several $\mathrm{GeV} / c^{2}$ with respect to the final results of the individual collaborations $[4,5,7,8]$ and the previous combination [3]. In the type II $2 \mathrm{HDM}$ scenario, assuming that the two decays $\mathrm{H}^{+} \rightarrow \mathrm{cs}$ and $\mathrm{H}^{+} \rightarrow \tau^{+} v$ exhaust the $\mathrm{H}^{+}$decay width, mass limits are obtained as a function of the branching ratio $\mathrm{Br}\left(\mathrm{H}^{+} \rightarrow \tau^{+} \nu\right)$. A $95 \%$ C.L. lower limit on the charged Higgs mass, independent 
LEP 183-209 GeV
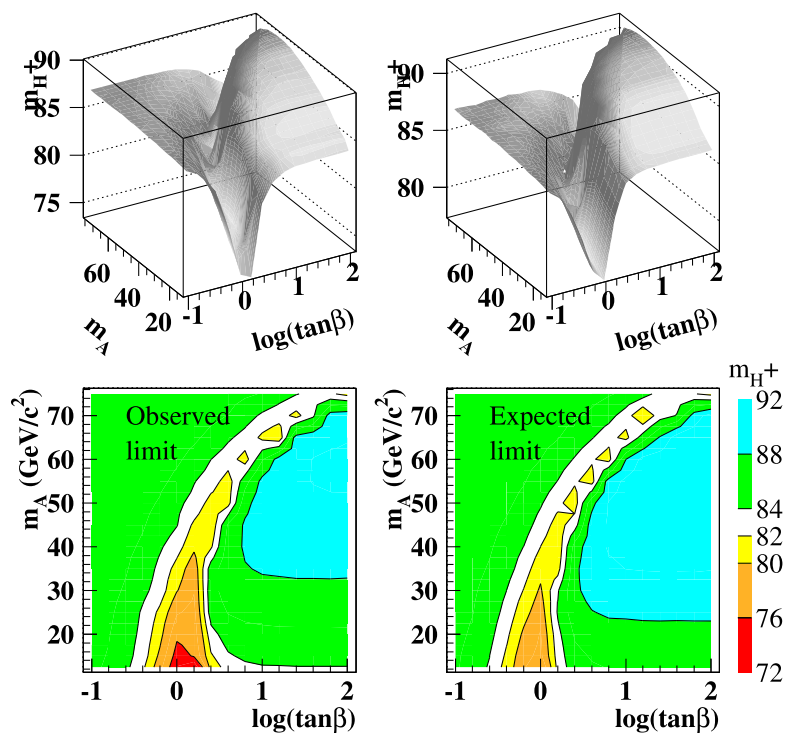

Fig. 7 Type I 2HDM: Observed (left) and expected (right) $95 \%$ C.L. lower limits on the mass of the charged Higgs boson. The color-mass correspondence is indicated on the right hand side (units are $\mathrm{GeV} / c^{2}$ )

\section{LEP 183-209 GeV}
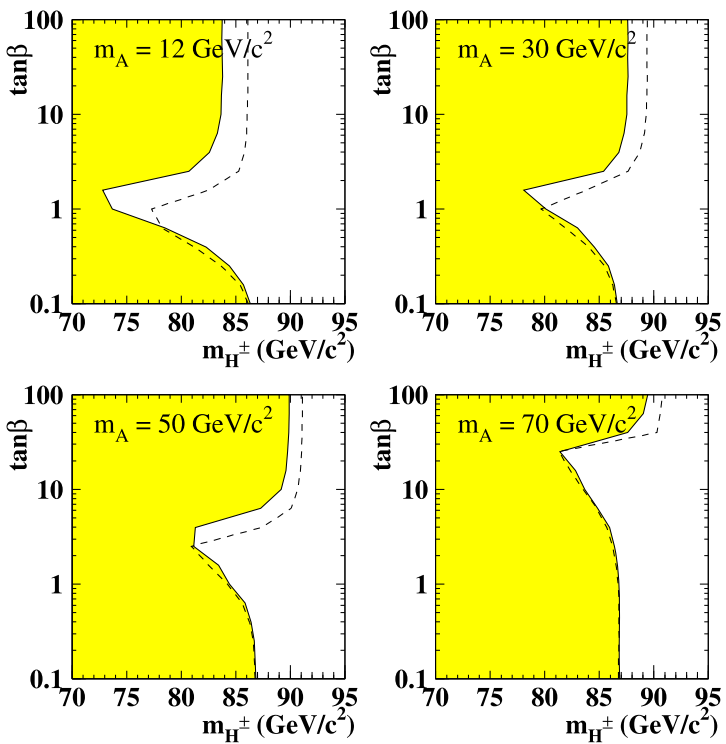

Fig. 8 Type I 2HDM: Excluded regions at $95 \%$ C.L. in the $\left(m_{\mathrm{H}^{ \pm}}\right.$, $\tan \beta$ ) plane for different values of $m_{\mathrm{A}}: 12,30,50$ and $70 \mathrm{GeV} / c^{2}$. The dashed line represents the expected exclusion limit

of its fermionic decay modes, is found to be $80 \mathrm{GeV} / c^{2}$. Thanks to analyses by DELPHI and OPAL in the bosonic $\mathrm{W}^{*} \mathrm{~A}$ decay channels, a new scenario, for type I $2 \mathrm{HDM}$, is also studied. In this case, masses of the charged Higgs bo- son below $72.5 \mathrm{GeV} / c^{2}$ are excluded at the $95 \%$ C.L. for A masses above $12 \mathrm{GeV} / c^{2}$.

Acknowledgements We would like to thank the CERN accelerator division for the excellent performance of the LEP accelerator in its high-energy phase. The LEP working group for Higgs boson searches would also like to thank the members of the four LEP experiments for providing their results and for valuable discussions concerning their combination.

Open Access This article is distributed under the terms of the Creative Commons Attribution License which permits any use, distribution, and reproduction in any medium, provided the original author(s) and the source are credited.

\section{References}

1. ATLAS Coll., Phys. Lett. B 716, 1 (2012)

2. CMS Coll., Phys. Lett. B 716, 30 (2012)

3. Search for Charged Higgs bosons: preliminary combined results using LEP data collected at energies up to $209 \mathrm{GeV}$. LHWG Note/2001-05, July 2001, and arXiv:hep-ex/0107031

4. A. Heister et al. (ALEPH Collaboration), Phys. Lett. B 543, 1 (2002)

5. J. Abdallah et al. (DELPHI Collaboration), Eur. Phys. J. C 34, 399 (2004)

6. P. Abreu et al. (DELPHI Collaboration), Phys. Lett. B 460, 484 (1999)

7. P. Achard et al. (L3 Collaboration), Phys. Lett. B 575, 208 (2003)

8. G. Abbiendi et al. (OPAL Collaboration), Eur. Phys. J. C 72, 2076 (2012)

9. G. Abbiendi et al. (OPAL Collaboration), Eur. Phys. J. C 7, 407 (1999)

10. The LEP Working Group for Higgs Boson Searches consists of members of the four LEP collaborations and of theorists among whom S. Heinemeyer is co-author of this paper

11. S.L. Glashow, S. Weinberg, Phys. Rev. D 15, 1958 (1977)

12. E.A. Paschos, Phys. Rev. D 15, 1966 (1977)

13. L.J. Hall, M.B. Wise, Nucl. Phys. B 187, 397 (1981)

14. A.G. Akeroyd, Nucl. Phys. B 544, 557 (1999)

15. The LEP Collaborations, the SLD Collaboration, the LEP Electroweak working group, the SLD Electroweak and Heavy Flavour Groups. Phys. Rep. 427, 257 (2006)

16. D. Decamp et al. (ALEPH Collaboration), Phys. Rep. 216, 253 (1992)

17. P. Abreu et al. (DELPHI Collaboration), Z. Phys. C 64, 183 (1994)

18. O. Adriani et al. (L3 Collaboration), Z. Phys. C 57, 355 (1993)

19. G. Alexander et al. (OPAL Collaboration), Phys. Lett. B 370, 174 (1996)

20. G. Ganis, P. Janot, The HZHA Generator: CERN Report 96-01, vol. 2, p. 309 (1996). Version 3, released in December 1999, https://janot.web.cern.ch/janot/Generators.html

21. A.G. Akeroyd et al., Eur. Phys. J. C 12, 451 (2000)

22. M. Skrzypek et al., Comput. Phys. Commun. 94, 216 (1996)

23. A. Denner et al., Phys. Lett. B 475, 127 (2000)

24. S. Jadach et al., Phys. Rev. D 61, 113010 (2000)

25. A. Ballestrero et al., Comput. Phys. Commun. 152, 175 (2003)

26. J. Fujimoto et al., Comput. Phys. Commun. 100, 128 (1997)

27. The LEP Collaborations, ALEPH, DELPHI, L3, OPAL, and the LEP Electroweak Working Group, Phys. Rep. (2013, submitted). arXiv:1302.3415 [hep-ex]

28. ALEPH, DELPHI, L3, OPAL Collaborations, The LEP working group for Higgs boson searches, Phys. Lett. B 565, 61 (2003) 
29. R.D. Cousins, V.L. Highland, Nucl. Instrum. Methods Phys. Res., Sect. A 320, 331 (1992)

30. The LEP collaborations, The LEP working group for Higgs boson searches, Eur. Phys. J. C 47, 547 (2006)
31. G. Abbiendi et al. (OPAL Collaboration), Eur. Phys. J. C 27, 311 (2003)

32. J. Abdallah et al. (DELPHI Collaboration), Eur. Phys. J. C 38, 1 (2004) 